\section{Response to: 'Do we need the PFAPA syndrome in adults with non-monogenic periodic fevers?' by Fayand et al}

We really thank Fayand and coworkers for their interest to the new Eurofever/PRINTO classification criteria for periodic fever, aphthous stomatitis, pharyngitis and cervical adenitis (PFAPA) ${ }^{1}$ and for their interesting exercise to apply the three most recent criteria to their population of adult patients with recurrent fever fulfilling the revised Marshall's criteria for PFAPA. ${ }^{2}$

Indeed, in the last few years, three new criteria for PFAPA have been proposed. ${ }^{3-5}$ Of note, the methodology used for the development of the three set of criteria was rather different, as is for the process of their validation in an independent population.

Cantarini's criteria were exclusively developed for adult-onset PFAPA patients. Seventy-four adult patients fulfilling the modified Marshall's criteria were compared with 62 patients with fever of unknown origin (FUO). A multivariate analysis identified the set of variables with the highest accuracy in distinguishing PFAPA from FUO patients. ${ }^{4}$ The criteria have not been validated in an independent population, so far.

Vanoni's criteria were created using the standard consensus procedures (Delphi survey and Nominal Group Technique in a Consensus Conference) among 22 paediatric experts in autoinflammation. The new proposed criteria were tested in 80 paediatric PFAPA patients followed in two centres for autoinflammatory diseases (Genoa and Lausanne). For this validation process, the diagnosis of PFAPA was not done on the basis of modified Marshall's criteria, but on the clinical judgement of the two centres. Notably, only $51 \%$ of the patients fulfilled the new criteria. ${ }^{5}$

The new Eurofever/PRINTO) criteria were developed with a multistep evidence-based approach. A Delphi questionnaire survey was proposed to 162 international experts in autoinflammatory diseases to identify the best possible candidate classification criteria for PFAPA syndrome. In the second step, 360 random paediatric and adult patients with recurrent fevers (familial mediterranean fever (FMF), TNF receptor 1 associated periodic syndrome (TRAPS), mevalonate kinase deficiency (MKD), cryopyrin associate periodic syndrome (CAPS), PFAPA and undifferentiated systemic autoinflammatory diseases (SAID)) from the Eurofever registry were evaluated by a panel of 25 clinicians and eight geneticists blinded to patients' diagnosis. Patients were classified on the consensus of at least $80 \%$ of the experts. In the third step, a multivariate statistical analysis performed on patients validated by the experts, identified for each condition a number of different set of variables able to discriminate them from the other confounding diseases. The best final classification criteria were chosen using the nominal group technique in a Consensus Conference that involved 33 international experts. Finally, all the new criteria, including those for PFAPA, were validated in an independent data set of 1018 patients extracted from the Eurofever registry not previously included in the statistical analysis. ${ }^{3}$

In our opinion, the strict methodological and evidence-based approach used for the development of the new Eurofever/ PRINTO classification criteria should indicate them as the most reliable criteria available so far either for children or adults.

It is important to note that in the validation phase, the accuracy of the new Eurofever/PRINTO PFAPA criteria was $81 \%$, with an high specificity (98\%) and a much lower sensitivity $(66 \%))^{3}$ This discrepancy between sensitivity and specificity was expected, since classification criteria are essentially done to identify patients with a very high probability to suffer from a given disease to be included in clinical trials or translational studies. Indeed, the classification criteria should not be used in the daily clinical practice and should not be considered as diagnostic criteria for which pathognomonic criteria are indeed necessary.

The different performances of the three classification criteria in the population analysed by Fayand et al could be merely due to patient selection methodology used in their study. ${ }^{1}$ As stated above, patients were selected according the modified Marshall's criteria. However, previous studies have provided evidence of the low accuracy of these latter criteria when applied to a population of patients with different forms of recurrent fevers, including the monogenic ones. ${ }^{6}$ Furthermore, the studied population seems to represent a mix between PFAPA patients with classical onset, as young child and adult-onset PFAPA patients. Since the phenotype may change with the time, it would be interesting to compare the classification obtained considering the initial clinical picture of the patients or that present at the time of the adult consultation. In table 1 , we notice that the median age of onset was between 2.5 and 5 years in patients fulfilling the PRINTO and Vanoni criteria, 21.5 years for the Cantarini criteria and 7 years for the not classified patients.

On the basis of the high variability in the performance of the different PFAPA criteria observed in their study, Fayand and coworkers postulate the hypothesis that PFAPA syndrome should not be considered as a separate entity in adults presenting with recurrent fever episodes, that should be classified as undefined systemic autoinflammatory diseases (USAID). To support their thesis, the authors provide, in their study, the evidence that almost $50 \%$ of their patients had a complete or partial response to colchicine independently from the satisfaction on any of the three classification criteria. However, it should be noted that the higher rate of response to colchicine $(72 \%)$ was observed in patients not classified by any of the three criteria, while the lower rate of response to colchicine (43\%) was observed in those fulfilling the Eurofever/PRINTO criteria.

On the other hand, it is also clear that a relevant percentage of patients display the same response to steroids on demand observed in the paediatric PFAPA patients and that four out of 11 patients who underwent tonsillectomy had a complete response. These observations clearly show the extreme heterogeneity in these groups of patients with idiopathic, not-monogenic, recurrent fevers.

In two recent studies, the term of SURF (systemic undefined recurrent fevers) was proposed, with the actual aim to identify a more homogeneous group of patients with recurrent fevers characterised by a minor pharyngeal and lymph node involvement, an higher prevalence of arthritis and skin rash, and a good response to colchicine and anti-IL-1 blockers. ${ }^{78}$ At least in children, this subgroup of patients should be clearly distinguished from the more common PFAPA patients.

It is also conceivable that a small percentage of adult patients with recurrent fevers could present the same clinical features of paediatric PFAPA deserving an appropriate treatment and follow-up.

In our opinion, the available classification criteria should be used to set up longitudinal studies enrolling paediatric and adult patients with recurrent fevers in order to verify the existence of different conditions (for clinical presentation, follow-up and response to treatment) in the heterogeneous group of USAID commonly observed in the daily clinical practice.

Marco Gattorno $\odot,^{1}$ Michael Hofer, ${ }^{2}$ Federica Vanoni, ${ }^{3}$ Silvia Federici, ${ }^{4}$ Luca Cantarini, ${ }^{5}$ Nicolino Ruperto, ${ }^{6}$ for the Paediatric Rheumatology International Trials Organisation (PRINTO) and the Eurofever Registry 
${ }^{1}$ Istituto Giannina Gaslini, Genova, Italy

${ }^{2}$ Department of Paediatrics, Centre Hospitalier Universitaire Vaudois (CHUV), Lausanne, Switzerland

${ }^{3}$ Ospedale Regionale di Bellinzona e Valli, Bellinzona, Switzerland

${ }^{4}$ Division of Rheumatology, IRCCS Ospedale Pediatrico Bambino Gesù, Roma, Italy

${ }^{5}$ Interdepartmental Research Center of Systemic Autoimmune and Autoinflammatory Diseases, University of Siena, Siena, Italy

${ }^{6}$ Pediatria II—PRINTO, IRCCS G Gaslini, Genova, Italy

Correspondence to Marco Gattorno, Istituto Giannina Gaslini, Genova 16147, Italy; marcogattorno@gaslini.org

Correction notice This article has been corrected since it published Online First. The PRINTO and Eurofever Registry author details were added.

Handling editor Josef S Smolen

Contributors MG, MH, FV, SF, LC and NR drafted the letter and approved the final version.

Funding This study was funded by Novartis and SOBI, E-rare-3 project (INSAID, grant 003037603), Executive Agency For Health and Consumers (Eurofever, Project No 2007332)

Competing interests None declared.

Patient consent for publication Not required.

Provenance and peer review Commissioned; internally peer reviewed.

(c) Author(s) (or their employer(s)) 2020. No commercial re-use. See rights and permissions. Published by BMJ.

\section{A) Check for updates}

To cite Gattorno M, Hofer M, Vanoni F, et al. Ann Rheum Dis Epub ahead of print: [please include Day Month Year]. doi:10.1136/annrheumdis-2019-216862
Received 25 January 2020

Accepted 27 January 2020

\section{Linked}

http://dx.doi.org/10.1136/annrheumdis-2019-216827

Ann Rheum Dis 2020;0:1-2. doi:10.1136/annrheumdis-2019-216862

\section{ORCID iD}

Marco Gattorno http://orcid.org/0000-0003-0704-1916

\section{REFERENCES}

1 Fayand A, Hentgen V, Ducharme-Bénard S, et al. Do we need the PFAPA syndrome in adults with non-monogenic periodic fevers? Ann Rheum Dis 2019:pii: annrheumdis-2019-216827.

2 Thomas KT, Feder HM, Lawton AR, et al. Periodic fever syndrome in children. J Pediatr 1999;135:15-21.

3 Gattorno M, Hofer M, Federici S, et al. Classification criteria for autoinflammatory recurrent fevers. Ann Rheum Dis 2019;78:1025-32.

4 Cantarini L, Vitale A, Sicignano LL, et al. Diagnostic criteria for adult-onset periodic fever, aphthous stomatitis, pharyngitis, and cervical adenitis (PFAPA) syndrome. Front Immunol 2017:8:1018.

5 Vanoni F, Caorsi R, Aeby $\mathrm{S}$, et al. Towards a new set of classification criteria for PFAPA syndrome. Pediatr Rheumatol Online J 2018;16:60.

6 Gattorno M, Caorsi R, Meini A, et al. Differentiating PFAPA syndrome from monogenic periodic fevers. Pediatrics 2009;124:e721-8.

7 Papa R, Rusmini M, Volpi S, et al. Next generation sequencing panel in undifferentiated autoinflammatory diseases identifies patients with colchicine-responder recurrent fevers. Rheumatology 2019;28:pii: kez270.

8 Ter Haar NM, Eijkelboom C, Cantarini L, et al. Clinical characteristics and genetic analyses of 187 patients with undefined autoinflammatory diseases. Ann Rheum Dis 2019;78:1405-11 